\title{
Non-Recursive Relations Structure-Functionality
}

\author{
Maria K. Koleva \\ Institute of Catalysis, Bulgarian Academy of Sciences, Sofia, Bulgaria \\ Email: mkoleva@bas.bg
}

Received 4 January 2016; accepted 20 March 2016; published 23 March 2016

Copyright (C) 2016 by author and Scientific Research Publishing Inc.

This work is licensed under the Creative Commons Attribution International License (CC BY).

http://creativecommons.org/licenses/by/4.0/

\begin{abstract}
Non-recursive relations structure-functionality is an exclusive property of the recently introduced concept of boundedness. They provide a leading role of the hierarchy of the functional organization in the evolution of each and every complex system. The novel evolution strategy appears as a counterpart rather than as opponent to the survival of the fittest strategy because the survival of the fittest is more advantageous strategy in a slow varying environment while the novel strategy is more advantageous for a rapidly changing environment. Alongside, the non-recursive relations structure-functionality serves as grounds for coexistence of scaling dependent and scaling independent properties of complex systems.
\end{abstract}

\section{Keywords}

Boundedness, Evolutionary Strategy, Power Laws, Fractals, Action, Heisenberg-Like Relations

\section{Introduction}

The study of complex systems is a rapidly developing field of interdisciplinary science. The major interest in this field is provoked by the well-established fact that complex systems behavior has certain properties which are still unexplained in a self-consistent way. My goal now is to add credible arguments that these problems are so serious and fundamental that their successful explanation require a novel explanatory paradigm.

Let me start with the notion of a complex system: it is how parts of a system are organized so that the system behaves as a single object and how it interacts with its environment. Thus, the notion of a complex system encompasses an enormous variety of systems ranging from physical ones such as quasar pulsations, to biological such as DNA sequences, to social ones such as financial time series. Yet, the most remarkable property is that intelligent systems such as ant colonies, music and speech also belong to the class of complex systems. In this respect, a striking example is that a Beethoven symphony, a product of an ingenious mind, and the traffic noise, also of human origin, but quite unintelligent in its appearance, share the same characteristic. 
The most puzzling property of complex systems is posed by the ubiquitous coexistence of specific and universal properties shared by each and every system. Thus, though all mammals share the same universal properties, a trained physician recognizes whether a given heartbeat record comes from a human or cat. Moreover, he can distinguish the individual heartbeat of each cat living in the world.

Further, there are two types of specific properties of complex systems: scaling dependent and scaling independent. The scaling independent properties are self-similar and their functionality is subject to the so called allometric scaling. An example is the vascular system of all biological species [1] [2]. The scaling dependent properties are shapes and size of the arms, legs, heads, etc. They are highly specific for each kind of objects and changes substantially with the change of scale.

The major dilemma is whether the above classification of properties is a product of structural differentiation only or it commences from a highly non-trivial interplay between structural and functional properties. The first alternative commences from the traditional reductionist approach based on the general assumption that the relation structure-functionality is expressed by recursive means only. In turn, this renders a leading role of the structure optimization for achieving the best functionality. The most famous example of that setting is the theory survival of the fittest which asserts that the most fitted structure gives rise to the best functionality. And the most fitted structure is the result of specific optimization of the structure.

However, this approach suffers the following fundamental flow: the process of optimization is self-organized only in the setting of algorithmic intelligence [3]. This is because the self-organized optimization is made by means of Random Boolean Networks whose major property is to reproduce the formal logic on each and every hierarchical level. More about this can be found in Section 2. However, all living organisms respond in a way different from formal logic. The following general properties are exclusive for the response of living organisms:

- The response does not follow silently the impact. Put in other words, there is no single law which all species of a kind to follow and which is a single match to a given impact.

- The systems are able to autonomous creation and comprehension of information. To remind that formal logic needs an external mind for both creation and comprehension of information.

- The human intelligence is able to discriminate true from false statements on the contrary to formal logic which is not able to discriminate between true and false statements.

Further I called these properties semantic intelligence.

The dilemma is whether the semantic intelligence is the result of specific structural peculiarities or one should adopt a novel paradigm for their explanation where the functionality acquires new role. I adopt the second alternative by means of assuming that the relation structure-functionality must be non-recursive. Non-recursive relation structure-functionality naturally arises in the setting of the recently put forward by me concept of boundedness [4]. One of the major outcomes of the non-recursivity of the relation structure-functionality is that the semantic intelligence explicitly utilizes it for setting the properties listed above. Another exclusive for the concept of boundedness property is that the survival strategy of each complex system is determined by its functional properties rather than the structural ones [5]. In turn, this renders a leading role of the semantic intelligence in the evolution of the complex systems.

The major goal of the present paper is to demonstrate that:

- Different parts of a complex system behave as autonomous objects and thus to explain the coexistence of the scaling dependent and scaling independent properties.

- To demonstrate that the coarse-grained behavior of all complex systems is universal although it comes from different structures.

The exclusive properties of the universal coarse-grained behavior open the door to a new route to evolution of complex systems.

The paper is organized as follows: The proof that Random Boolean Networks obtain only algorithmic intelligence is presented in Section 2. The basic principles of the concept of boundedness and of the semantic intelligence are presented in Section 3. The proof that different parts of a complex system behave as autonomous objects is presented in Section 4. The proof that the coarse-grained behavior of all complex systems is universal is presented in Section 5.

\section{Random Boolean Networks and Algorithmic Intelligence}

Random Boolean Networks (RBN) are known as $N K$ networks or Kauffman networks. An RBN is a system of $N$ 
binary-state nodes (representing genes) with $K$ inputs to each node representing regulatory mechanisms. The two states (on/off) represent respectively, the status of a gene being active or inactive. The variable $K$ is typically held constant, but it can also be varied across all genes, making it a set of integers instead of a single integer. In the simplest case each gene is assigned, at random, $K$ regulatory inputs from among the $N$ genes, and one of the possible Boolean functions of $K$ inputs. This gives a random sample of the possible ensembles of the $N K$ networks. The state of a network at any point in time is given by the current states of all $N$ genes. Thus the state space of any such network is $2^{N}$. The major property of every RBN is that, on fine-tuning of the input, it reaches an attractor where it stays for arbitrary long time. The remarkable property of that state is its robustness to local failures. In turn the latter makes it very attractive for developing a promising hardware which is insensitive to local failures.

RBNis an example how a network whose elements (nodes) are subjects to formal logic reproduces this property atthe next hierarchical level. Indeed, each node could reside in one of two states (active or inactive) and switches between them under the command "IF": "IF" the neighborhood impact reaches certain threshold, the node switches its state. The same is with the attractors: "IF" the local concentration of the nodes in certain state reaches certain value, the system switches to another attractor. Thus, the Boolean networks indeed reproduce formal logic in a way that makes them much more diverse in their abilities and much more reliable in their operations, properties which render them an elaborate hardware for Turing machines.

The importance of the Boolean networks for us lies in the following: the invariance of the formal logic as a type of response which appears under self-organization is remarkable!

\section{Basic Principles of the Concept of Boundedness}

The major principles of the concept of boundedness and the semantic intelligence are considered in the present section. The explicit role of semantic intelligence in the determination of the efficiency of the survival strategy is considered in sub-Section 3.3.

Next the grounding assumptions of the concept of boundedness are presented:

- A complex system remains stable if and only if the rate and amplitude of variations that it exerts in response to an ever-changing environment are bounded to specific for the system margins.

- The response is local and not pre-determined. This implies that it depends on the current state of a system and the current environment impact. Yet, in order to sustain boundedness of rates it behaves in a non-linear and non-homogeneous way. Non-homogeneity of the response replaces the traditional idea of linear superposition of the responses each of which come as response to a given component of the current environmental impact independently from the response to the other components. Linear superposition of response is abandoned since it does not provide local boundedness even if each of the current partial responses does. Put in other words, the sum cannotprovide boundedness although each of the participating terms provides it. Also, the sum can provide boundedness while some of the components do not, i.e. sum of vectors.

- Complex systems are self-organized in a hierarchy of responses so that different hierarchical levels are linked through inter-level feedbacks. An exclusive property of the inter-level feedbacks is that each of them operates as a bounded irregular environment for the self-organization at each and every hierarchical level. In turn this provides bi-directionality of the hierarchy as it goes both bottoms up and top down. This is in sharp contrast with the traditional approach where different objects are considered closed systems and the hierarchy goes only bottom up.

A central for the boundedness result is the proof that there exists a presentation basis where the response to an unspecified ever-changing environment decomposes into two parts, specific and universal one, each of which has characteristics that are robust to environmental variations. The rigorous assertion is that the power spectrum of each time series that represents the behavior of a complex system is decomposable into two parts: a specific discrete band and a continuous band whose shape is universal. Further, the claim is that the decomposition is additive and it happens with constant in the time accuracy.

In addition to these two parts in the power spectrum a non-recursive component persists. It commences from the highly non-trivial interplay between the specific and the universal part. Since each time series which represents a record of the behavior of a complex system is bounded irregular sequence, it could be presented as wild and permanent bounded "twisting" and "winding up" around a "skeleton" built on the discrete band of the power spectrum. Thus, the motion resembles the motion on a torus with irrational frequencies. In the present 
case the motion never stops and thus the extra-line associated with irregular motion around the skeleton appears always as non-recursive regardless to the relations among the thresholds over the corresponding quantities and the other scales in the corresponding system.

The greatest value of that decomposition is:

- It allows unambiguous separation of an object from its environment. The notion of an object consists of specific pattern, called homeostasis because its characteristics remain intact in an ever-changing environment. Moreover, one can define them regardless to the details of that environment.

- Although the exact local behavior remains unpredictable, it provides predictability of the behavior of each complex system up to the predictability of its current homeostatic pattern.

- The persistence presence of a non-recursive component constitutes the non-recursive way of relating structural and functional properties. Indeed, while structural properties are defined through the specific pattern whose power spectrum is given by the discrete band, the functional properties are presented through the entire power spectrum, i.e. through the interplay among specific, noise and non-recursive component altogether.

\subsection{Semantic Intelligence as a Sub-Class of Complex Systems Behavior}

Next the necessary conditions for the realization of the semantic intelligence are considered. It should be stressed that the class of intelligent complex systems is a sub-class of complex systems. Thus, all complex systems are subject to boundedness but the intelligent complex systems are subjected to an additional constraint which will be presented next.

An exclusive property of any complex system considered in the frame of boundedness is that its state space is partitioned into domains so that each domain is characterized by a specific for it homeostatic pattern; the rate of intra-domain motion is bounded and irregular. In result the motion among domains is restricted to the adjacent domains only. The boundedness of the inter-domain motion renders the state space trajectories to be non-random. This is so because any trajectory in a state space goes in such a way that it jumps only to adjacent states. The allowed inter-domain motion is confined only to the adjacent domains next to any given domain. The selection of any of the adjacent domains is random one since in each run it depends on the current intra-domain trajectory which is irregular.

The general necessary constraint which provides implementation of the semantic intelligence by physical processes consists of requirement that the state space of any intelligent complex system is to be partitioned into at least 4 domains. The reason behind the particular choice of no less than 4 domains will become clear in the next sub-section.

The utilization of this constraint is two-fold:

- The first assumption is to associate information symbols with intra-domain homeostatic patterns. The far-going consequence of that association consists of providing algorithmic uniqueness of each and every specific law. The algorithmic uniqueness is achieved through the ubiquitous presence of a non-recursive component additional to the homeostatic and noise characteristics. To remind, a "non-recursive" implies that neither specific law can be achieved by finite number of steps organized in an algorithm. The non-recursive component comes from a highly non-trivial interplay between the homeostatic pattern and inter-level feedbacks for every intelligent complex system as explained in the previous section. In turn, it makes different specific laws unique and algorithmically unreachable from one another. It should be stressed that the comparison with the traditional explanatory paradigm displays a sharp contrast. Indeed, the traditional explanatory approach assumes existence of a universal law such that all specific laws are its algorithmic derivatives.

- The second assumption consists of association of the meaning of each semantic unit with the performance of a specific non-mechanical engine built on the corresponding inter-domain orbit in the state space. As an example of non-mechanical engines may be considered the bio-chemical cycles in living organisms. The exclusive property of presentation of the meaning of a semantic unit through performance of an engine is that the latter provides sensitivity to permutations of the semantics. Indeed, like the semantics of human languages where the meaning of each word depends on the order of the letters in it, an engine performs differently if it operates in different directions. Thus, for example the famous Carnot engine operates in one direction as a pump and in the opposite direction it performs as refrigerator.

Thus, each semantic response consists of hierarchy of cycles which follow through adjacent states only irres- 
pectively to the intensity of the current environmental impact. In turn, this provides the most fundamental property of the semantic intelligence to be the autonomous creation and comprehension of information.

The fact that the admissible trajectories in the state space go only through adjacent states provides the property of the response not to follow silently the impact. Thus, the corresponding system organizes it response in a non-linear and non-homogeneous way so that to keep the homeostasis intact as long as possible.

Alongside, the fact that the admissible trajectories in the state space go only through adjacent states provides the property of semantic intelligence to discriminate autonomously between true and false statements. A 'true' statement corresponds to that unique trajectory, which has been physically realized in a given run; a 'false' statement is the one that follows an imaginary trajectory that comprises a non-admissible state; a counterfactual corresponds to an admissible trajectory which has not been realized in a given run. And last but not least, an improbable statement corresponds to an imaginary impasse: the statement does not correspond to any trajectory. Thus, the automatic selection of trajectories to admissible and non-admissible renders the fundamental difference of the present approach from the traditional algorithmic theory and the formal logic which are grounded on the assumption that all trajectories are available. Let us provide an example: the sentences "the Sun rises at east" and "the Sun rises at west" are both true in the setting of algorithmic theory because they are both grammatically correct; on the other hand in the setting of boundedness the first sentence "the Sun rises at east" is true since it corresponds to a physically realizable trajectory while the sentence "the Sun rises at west" is false because it corresponds to a physically un-realizable trajectory.

Outlining, the semantic intelligence which arises from the concept of boundedness has the empirical properties of the living organisms intelligence considered in the Introduction.

\subsection{Second Law and Semantic Intelligence}

The next point is the relation between physical processes which implement semantic intelligence and their environment. The importance of this question is rendered by the fact that in the traditional algorithmic approach no hardware is in balance with its environment. This is so because it is assumed that any hardware can execute any algorithm. In turn this opens the door to an uncontrolled "drainage" of the environment or its uncontrolled heating. Thus a question arises: can one built information perpetuum mobile? The answer is negative and it consists of reformulation of the Second Law so that its availability to encompass the widest scope of systems.

A clue for the most general formulation of the Second Law in the frame of boundedness is the need for its availability to semantic intelligence. Since the latter is executed by means of performance of non-mechanical engines, an appropriate formulation should be grounded on their generic properties. I have proved [4] that in the setting of the concept of boundedness, the efficiency of a non-mechanical engine never exceeds the efficiency of corresponding Carnot engine where the engine is free from necessity of a physical coupling to two heat reservoirs. Alongside, the proof is free from the condition for entropy maximization viewed as condition for reaching equilibrium. Thus the proof substantiates the most ubiquitous formulation of the Second Law to impose ban over perpetuum mobile.

It should be stressed that the present formulation of the Second Law justifies the general condition about the necessity of at least 4 domains with different homeostasis for implementation of semantic intelligence. Indeed, suppose that a cycle consists of a trajectory that goes through 3 domains only. Then the corresponding engine performs all possible permutations in a single cycle by means of running it in opposite directions and starting at different states. Thus the words "dog" and "god" and words 'on' and 'no' are product of the same engine but run in opposite direction. Therefore, it is obvious that only orbits which go through at least 4 states provide different cycles for different permutations. Further, the work produced by different engines is a measure for the efforts exerted behind any semantics. Since the engines are specific, the corresponding work is also specific for each semantic response. In turn this puts the hierarchical organization of the semantic response prior to the fact whether it is fastest or most efficient. This fact is opposite to the traditional algorithmic approach where the fastest computers and the most efficient algorithms are most valued.

It is worth noting the sharp contrast with the traditional approach. A generic property of the state space in the traditional approach is the Markovianty. It implies that successive jumps are not correlated and the trajectories never close. As a result no semantic intelligence is ever possible in the frame of the traditional approach.

The most fundamental property of the semantic intelligence, the autonomous creation and comprehension of information comes at a price: unlike a Turing machine which is able to execute every algorithm, the semantic 
intelligence executes only algorithms compatible with its "hardware". However, this is rather an advantage than a setback because it serves as grounds for autonomous discrimination of a true and a false statement by semantic intelligence. Then, the distinctive properties of both algorithmic approach and semantic intelligence make them rather counterparts than opponents.

Summarizing, the additional constraint to which intelligent complex systems are subject is that its state space must consists of at least 4 domains. This condition is satisfied for spatially heterogeneous systems only. The state space of homogeneous systems has only 2 domains. Put in other words, only systems which are self-organized in patterns or are subject to morphogenesis can exhibit semantic intelligence. Yet, at this point a question arises: the necessary condition for heterogeneity is to have 3 domains in the state space. Then, to what generic type of pattern formation the requirement of about at least 4 domains corresponds?!

A great advantage of the semantic intelligence is that it is in balance with the environment. This fact is an immediate consequence of the assumption about considering semantic intelligence as a sub-class of complex systems. Then any piece of semantics is executed as a sequence of information symbols. A generic property of this sequence is that, being a bounded irregular sequence, its variance is always finite [4].

\subsection{Semantic Intelligence and Survival Strategy}

Recently, I have proved [5] that the best survival strategy implies that a complex system is organized so that it spends minimum work on its interaction with the environment. The proof is strongly grounded on the central for the boundedness assumption that the response is executed in semantic-like manner whose implementation is constituted by operation of non-mechanical engines organized in a non-extensive hierarchy by means of grammar-like rules. The non-triviality of the obtained criterion is revealed through the inter-dependence between the size of the corresponding working cycle $L$ and the corresponding values of the thresholds of stability $\Delta$. The efficiency of the semantic response $\eta$, expressed through the ratio between the work associated with changes in the homeostasis and the energy spent on the interaction with the environment reads:

$$
\eta=L^{1-\frac{\log \Delta}{\log L}}
$$

This dependence appears as follows: any change in $L$ implies change in hierarchical structuring since the setting of basins-of-attraction at each level is controlled by neighboring levels through inter-level feedbacks. In turn, any change of $L$ is related to a change in $\Delta$. The exclusive property of those changes is that they are not proportional to one another since the relation impact-response is non-linear and non-homogeneous. An immediate consequence is that the transformation of an object into a novel one happens in an allometric-like manner, i.e. parts of the same function that belong to different objects are not self-similar.

Another issue which demonstrates the high non-triviality of the intertwining between the size of a cycle $L$ and the thresholds of stability for the interaction with the environment $\Delta$ is that any attempt to isolate the cycle from the environment, i.e. putting $\Delta \equiv 0$ yields infinite efficiency. However, this immediately implies building a universal perpetuum mobile whose generic property is that its characteristics do not depend on $L$. Put in other words, the latter implies that all objects in the Universe are perpetuum mobile. Thus, it becomes clear that the interaction with the environment is crucial for diversification of the objects in the Universe.

The explicit dependence of the efficiency coefficient on the size of working cycle straightforwardly demonstrates the role which the hierarchy of the semantic response plays in determination of the survival strategy. It should be stressed that the semantic intelligence is a functional property of the response not a structural one. Thus, the organization of the semantic intelligence plays the overwhelming role in determination of the best survival strategy on the contrary to the survival of the fittest where the structural optimization has the principal role.

\section{Different Parts of a Complex System as Autonomous Objects}

The setting that a complex system responds in a semantic-like way calls for substantiating semantic units as autonomous objects. Here the autonomy implies existence of properties that characterize the functionality of the corresponding unit as a unique object. To certain extent, this requirement is similar to the notion of mass and energy in the traditional physics where these notions are basic characteristics of each and every object. The notions of mass and energy are central for the traditional statistical mechanics since their participation in the equations of motion is crucial for the understanding of the traditional physics. To remind briefly, the mass explicitly 
enters the equations of motion while the energy has broader use. In mechanics it is associated with the notion of Lagrangian so that the equations-of-motion to follow from its existence. The relation between the equation of motion and the Lagrangian are given by Euler-Lagrange equations whose origin is set by the Hamilton principle. The Hamilton principle asserts that a trajectory with fixed ends follows the one whose action is stationary.

Even though this result has been considered as one of the greatest results in the traditional mechanics, which strongly impacts all branches of modern physics, we cannot apply it without essential modification to the motion in a semantic state space. There is fundamental reason for this to be the case because, unlike the traditional motion, the motion in a semantic state space is not continuous and thus one cannot fix the ends of a trajectory with arbitrary accuracy. To remind, the trajectories in a semantic state space are bounded irregular sequences (BIS) which are first kind discontinuous curves in every point, i.e. at each point there is a jump of bounded size. Two questions arise: how far the trajectory could go and how close to a certain point it could pass. The answer to the first of these questions is: starting anywhere is the state space the trajectory could reach the thresholds of stability where it exerts a U-turn. My present task is to establish how close to a certain point it could pass and to find out the measures of this distance. Let me start with the observation that the boundedness sets the following inequalities at each point of the state space and for every trajectory. Let $\Delta x$ be the "jump" between any two successive states; $\Delta p$ to be the rate of that jump and $\Delta t$ to be the time interval at which the jump takes place. Then, since each of these quantities is bounded, we can define the following inequalities:

$$
\begin{aligned}
\Delta x_{i} \Delta x_{j} & \leq C_{x x} \\
\Delta x_{i} \Delta p_{i} & \leq C_{x p} \\
\Delta p_{i} \Delta p_{j} & \leq C_{p p}
\end{aligned}
$$

These relations very much resemble the famous Heisenberg relations in the quantum mechanics. To remind, the Heisenberg relations in the quantum mechanics set a relation between uncertainty of simultaneous measuring of the position and the velocity of a quantum object. This type of uncertainty comes out from the dualism particle-wave, a fundamental notion in quantum mechanics. The general conclusion that stems from the Heisenberg relations is that it is impossible to measure with finest precision both the position and the momentum of any quantum object.

Then, how we should understand the relations (2)-(4)? In the present case there is no particle-wave dualism. The answer is simple though non-trivial: we relate these uncertainty relations with the discontinuity of the motion. To compare, the motion in the traditional mechanics is everywhere continuous whereas in a semantic state space it is discontinuous in every point. The other ingredient for the uncertainty relations (2)-(4) is the boundedness: namely the boundedness sets the values of the constants $C_{i j}$. It should be stressed that without boundedness, no "uncertainty" relations of the above type are ever available.

However, the relations (2)-(4) comprise more than just setting the uncertainty of determining the position and the velocity in every moment. Indeed, let us define the following variable:

$$
\Delta S \equiv \Delta x \Delta p-\Delta E \Delta t
$$

which I put subject to the following constraint:

$$
\Delta S \equiv 0
$$

Thereby, I could call $S$ from Equations (5)-(6) "action”. And it is obvious that it retains the property of being stationary (expressed through Equation (6)) if and only if the variable $\Delta E$ is defined as:

$$
\Delta E=\frac{\Delta x \Delta p}{\Delta t}
$$

Note that $\Delta E$ from Equation (7) has the meaning of "energy" if defining "mass" as follows:

Defining:

$$
V=\frac{\Delta x}{\Delta t}
$$

and

$$
\Delta p=m V
$$


then:

$$
\Delta E=m V^{2}
$$

Thus, by means of defining stationarity of the "action", we simultaneously define "mass" and "energy" of the object that follows the trajectory of stationary action. Put it other way round I assert that the motion along a trajectory of a stationary action could be considered as a motion of well-defined single object whose constituents move coherently so that to preserve the covariance of the characteristics of the motion. Note, that though dependent on the position in the state space, neither the "mass" nor the "energy" selects a special time or space point along a stationary action trajectory. Thus, the stationary action trajectories still retain the major property of any other trajectory in a semantic state space, namely the property not to select and/or signals out any specific time and/or space point.

Further, it is important to stress on the non-extensivity of the above defined mass and energy. Note, that here neither of them is reducible to sum of mass/energy of the constituents! On the contrary, each of them is explicitly related to non-extensive collective variables characterizing the system. The important point is that defined through stationarity of the action, each of them serves as a characteristic of a motion of a single object. This property serves as grounds for substantiating the idea of a "module" as a functional unit, which, though being itself comprised of sub-units, behaves as a single object. Then, the notions of mass and energy serve as measures of differentiation of the modules one from another.

It should be stressed that the notion of action in the present context does not imply any extreme properties of motion in the state space. To compare, the traditional notion of action relates its stationarity with the extreme properties of the system, such as the principle of least action, minimum energy etc. Thus, the release of the notion of stationarity of the action from extreme properties opens the door to have many trajectories that fulfill the property of stationarity. Thus it is to be expected diversity of response, i.e. diversity of semantics. A fundamental value of the diversity of response is it s fault tolerance. Indeed, multi-valuedness of the response provides alternative "routes" for response in case of structural and/or functional damages.

It should be stressed that, on the contrary, the association of the stationarity of the action with the extreme properties most probably selects a single available trajectory. Thereby it is not to be expected manifestation of any diversity and non-extensivity of any response. In turn, the response is not fault tolerant in the traditional scenario.

Before elucidating the role of the presently involved characteristics of the motion with respect to the characteristics of highly organized semantics, let me draw some attention to the relations (2)-(4) and Equation (9). Indeed, the boundedness expressed through Equations (2)-(4) sets boundedness over the velocity $V$ and to the mass $m$. Then, Equation (10) could be presented in the following form:

$$
\Delta E \leq m C^{2}
$$

where $C$ is the maximum constraint over the velocity along a trajectory of a stationary action. It is obvious that Equation (11) surprisingly exactly matches the famous Einstein relation between mass and energy. My aim now is to focus the attention on the differences with the Einstein formula. The first one is that the bound $C$ is specific to every process (system) and so it does not set any universal constant unlike the case with the Einstein relation which put the light in a special position among all process in Nature. The second fundamental difference between the Einstein relation and Equation (11) is that, though Equation (11) quantifies transformations between "mass" and "energy", it happens under the constraint of keeping each of them bounded. On the contrary, the Einstein relation allows the mass to grow unrestrictedly when the velocity approaches the speed of light. Indeed, the famous relations $E=m C^{2}$ where $m=m_{0} / \sqrt{1-v^{2} / c^{2}}$ allows unrestrained growth of both energy and mass when the velocity $v$ approaches the speed of light $c$. However, since this relation is supposed universal, i.e. available for every system regardless to its particularities and since infinite energy means infinite work for its achievement, does the acceleration process which substantiates this relation implies involving of a special kind of "perpetuum mobile"? This line of suggestions and the relation between Equation (11) which, on the contrary, allow transformations of only bounded amounts of energy and mass, and the idea of ban over the perpetuum mobile is considered in section 3.2.

Outlining, I have demonstrated that every complex system consists of moduli which behave in a rather autonomous way. Their exclusive property is that their functional characteristics (mass, energy) are non-recursively 
related to the corresponding characteristics of their constituents. Note that the requirement about stationarity of action renders the properties of different moduli to vary substantially with the change in scale. In turn, the above considerations substantiate operational protocol for providing scaling dependent properties of complex systems.

\section{Universality of the Coarse-Grained Behavior}

The goal of the present section is to outline a general operational protocol for providing scaling independent properties of complex systems. As it will become clear this comes along with the universality of the coarsegrained behavior. It will be demonstrated that the target general operational protocol is explicitly related to persistent dynamical structure which strongly resembles fractals. However, unlike the traditional fractals where the repetition of the structure is scale-free and thus the notion of a fractal is inconsistent with the formation of any scaling dependent moduli and the notions of average and variance, in the context of boundedness fractality is coherent both with the formation of scaling dependent moduli and the notions of average and variance of any pattern that represents complex system behavior.

Let me start with a reminder about what a fractal is. Fractality implies that the spatial and temporal shape of the objects that represent complex system follows a general geometrical pattern which has the following property: the objects appear as a hierarchical structure which consists of repetition at different scale levels of the same basic building block. For example, the coast of Norway appears as hierarchical structure of fiords, and fiords within fiords, and fiords within fiords of fiords.

However, there is no an easy and straightforward answer to the question how long the coastal line is. The problem is that the length of the coastal line would depend on the ruler used for its measurement. One way to estimating the length of the coastal line is to measure how many boxes of certain size $\delta$ are necessary to cover the coast. If the coastal line were a straight line of length $L$, the number of boxes would be proportional to $\delta$, so that the measured length would be independent of $\delta$. Then, the length of the coastline would be independent from the size of the ruler. However, the number of boxes needed grows much faster than that since the boxes must follow the wrinkles of the coastline. It is obvious that the number of boxes strongly depends on the size of the ruler: the smaller the size, the more wrinkles are involved. In turn, the larger the length of the coastal line is.

The fact that the length of a fractal strongly depends on the ruler suggests conflict with stability of a system. In the present context stability implies that each system has well defined characteristics, length included. This conflict poses the following fundamental question: Is there any general dynamical pattern which renders the above discussed hierarchy of self-similarity that at the same time preserves the specific properties of any system? The answer to these question is positive but in the frame of the concept of boundedness.

The general dynamical pattern which gives rise to fractality lies in the general structure of the bounded irregular sequences which represent the structure of each object. It should be stressed that the time series follow the same general structure: the signal can be seen as a superposition of bumps of all scales and durations. The question is whether there is a general rule for this superposition. The answer is positive and it comes as follows: the coarse-grained structure of each bump can be considered as a fractal Brownian walk whose trajectory forms an excursion. An excursion consists of a trajectory of walk originating at a given point at moment $t$ and returning to it for the first time at the moment $t+\Delta$. The excursions are an immediate result of boundedness of rates and the requirement that the motion is confined within specific margins. Thus, the boundedness of the rates "keeps" the distance between successive steps within a given margins. In result, distant steps turn correlated. It should be stressed on the fact that these correlations are not a result of specific physical process but they are purely of mathematical origin. Then, it becomes clear the consistence between the boundedness of rates and the consideration that a sequence of excursions forms a homogeneous process such that different excursions participate as independent events. Indeed, each excursion due its specific shape to the boundedness of the rates. Alongside, each excursion is embedded in specific interval so that no other excursion of that or larger size is embedded in it. Thus, on return to the initial value, the corresponding trajectory follows through the embedding interval of that excursion since no other excursion of greater size can be embedded in it. On the other hand, after the excursion is completed, another one can start. It is obvious that both excursions are independent from one another. The only constraint imposed on the size of excursions is imposed by the thresholds of stability.

The embedding is a property introduced by the boundedness and has no analog for the unbounded sequences. It implies that each excursion is embedded in a larger interval whose duration is interrelated with the duration of the excursion itself. The major role of the embedding is that it does not allow overlapping of the successive ex- 
cursions and thus prevents growing of the excursion amplitude to arbitrary size. It results in permanent preservation of both boundedness of amplitude and boundedness of rates.

The characteristics of each excursion are amplitude $A$, duration $\Delta$ and embedding interval $T$.

The above picture (Figure 1) serves as a basic building block for the hierarchy of any fractal pattern since it is invariant under the operation of coarse-graining. The operation of coarse-graining consists of any operation such as local averaging, filtering etc. which acts in a non-linear and non-homogeneous way on the condition of leaving boundedness intact. This general result can be found in Chapter 5 of the book [4].

Thus, figuratively speaking, the excursions "protrude" out of the embedding interval and this happens at each scale level of the system up to the thresholds imposed by the boundedness. This is exactly what fractals do: each fractal "protrudes" out of its "embedding" interval and this happens at each scale level.

It is worth noting that the above discussed general dynamical pattern appears as a universal operational protocol not as law since in each concrete case the properties of the corresponding excursions are highly specific and set by the concrete dynamics of the corresponding system. On the other hand, what the general protocol does is to "embed" each excursion in a specific interval so that no other excursion of the same and larger size to be embedded in it.

The idea that the coarse-grained structure of each and every BIS consists of embedded excursions which form a homogeneous process implies that the frequency of occurrence of an excursion of size Ais time-independent and reads:

$$
P(A)=c A^{\alpha(A)} \frac{\exp \left(-A^{2} / \sigma^{2}\right)}{\sigma}
$$

The required probability $P(A)$ is given as a product of the term $A^{\alpha(A)}$ which is the statistical weight of a fractal consisting of an excursion of amplitude $A$ and its embedding interval, and the probability for appearance of excursion of that size (normal distribution); $\sigma$ is the variance of the BIS; $c=1 / \sigma^{\alpha(\sigma)}$ is the normalizing term. It is worth noting that the statistical weight of a fractal of size $A$ is given by the extension of the corresponding fractal because this is the interval where the fractal behaves as a single object. Thus, the extension of a fractal is equal to its length where the exponent $\alpha(A)$ is its Hausdorff dimension. The homogeneity of the excursion appearance ensures that $P(A)$ has the same value at every point of the sequence. It is worth noting that the Gaussian-like term $\frac{\exp \left(-A^{2} / \sigma^{2}\right)}{\sigma}$ in Equation (12) commences from the fact that the coarse-grained structure of any BIS is again BIS; then, according to the Lindeberg theorem [6] it has well-defined mean and variance and thus it is subject to normal distribution.

It is worth noting that the distribution (12) is universal and comprises average and variance, though it commences from fractal-like dynamical pattern which appears as stochastic sequence of embedded excursions.

A very important fact is that the distribution (12) has a fat tail which is excellently fitted by a power law with scaling dependent exponent. This excellent fit is most pronounced when the probability of the excursion is expressed through the following relation:

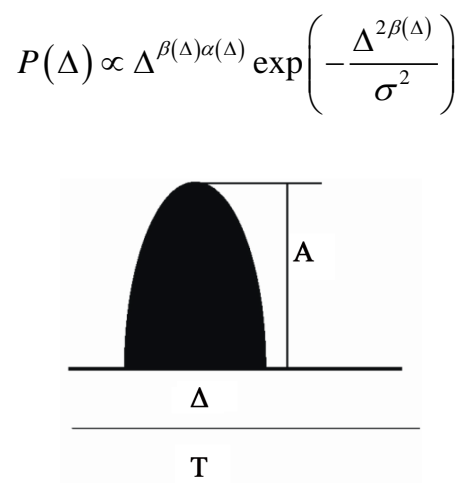

Figure 1. An excursion and its embedding interval. 
where the duration $\Delta$ of an excursion of size $A$ appears as an independent variable, namely it represents the length of the time window. Note that the size of an excursion $A$ is related to its duration $\Delta$ by the following relation: $A \propto \Delta^{\beta(\Delta)}$.

A plot of $P(\Delta)$ with $\alpha=1, \beta=0.3$ and $\sigma=2$ is presented in Figure 2 (continuous line). The heavy tail is fitted by the power function $f(\Delta) \propto \Delta^{\gamma(\Delta)}$ with scaling dependent exponent $\gamma(\Delta)=-0.1-0.005 \Delta$ (dotted line). The most impressive result of this approximation is that the surprisingly good fit is spanned over two orders of magnitude and covers the entire tail of the distribution $P(\Delta)$.

The universality of the probability for an excursion and the excellent fit of its fat tail with a power law suggest a coherent explanation of the ubiquity of the empirically observed fat tail distributions. The greatest advantage of the present considerations is that they are free from the problems which scale-free behavior poses. Indeed:

- The fractal-like behavior considered above is consistent with the formation of specific scaling dependent moduli. Thus, both scaling-independent and scaling dependent moduli can coexist.

- The fractal-like behavior considered above is consistent with the notions of average and variance.

- The fractal-like behavior considered above is consistent with the idea of semantic intelligence.

Next I will consider the crucial role of the fact that the best fit of the fat tail is provided by a power law with scaling-dependent exponent. The presence of scaling dependent exponent renders scaling dependent properties to be spread over a few scales only and gradually to modify into scaling dependent ones.

Yet, the more fundamental role of the universality of the distribution (12) is that it constitutes margins over the diversity of the individual response: it should vary so that on coarse-grained level to fit the above universality. Thus, the behavior of each and every subject has two components: the first one is specific individual response and the second one is the universal one whose dependence on the scale is characterized by the corresponding scale dependent exponent. The specific role of the variability of the individual response is to provide fault tolerance while the role of the universal component is to restrain the variability admissible individual responses within specific margins. In turn this renders a novel route to evolution where changes in the functionality acquire the leading role. Note that the corresponding structural changes are not subject of optimization of the type considered in section 2 but appear as a highly non-trivial interplay between structure and functionality where the hierarchical organization of semantic responses plays the leading role. It is worth reminding two things:

- The hierarchical organization of semantic response naturally commences from the non-recursivity of the relation structure-functionality which thus stands as the most fundamental reason for the above mentioned root to evolution.

- The scaling dependent exponent implies that the corresponding coarse-grained laws gradually changes with the scale. Thus, for example Kleiber law " $3 / 4$ " for metabolic scaling is relevant for species of larger mass but on decreasing the mass of species it approaches a scaling exponent of " $2 / 3$ ". However, although there is a smooth crossover between both scaling exponents, they imply fundamentally different mechanisms of metabolism.

Outlining, the novel evolution strategy appears as a counterpart rather than as opponent to the survival of the fittest strategy. Indeed, the survival of the fittest is more advantageous strategy in a slow verifying environment, the novel strategy is more advantageous for a rapidly changing environment.

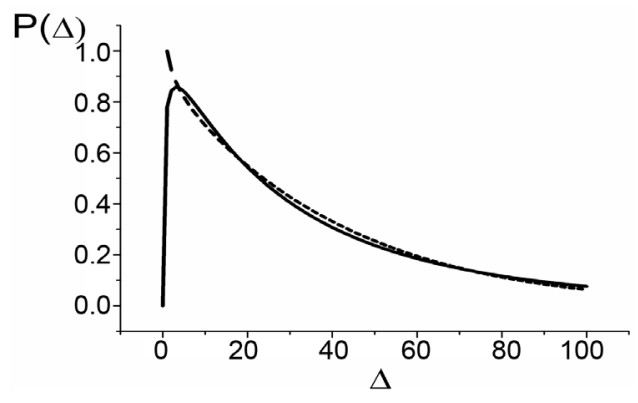

Figure 2. Approximation of the probability for an excursion (continuous line) with power function (dotted line). 


\section{Conclusions}

The most fundamental property of the recently introduced concept of boundedness consists of non-recursive relations structure-functionality. The impact of this property is persistent and encompasses the whole range of properties of the behavior of complex systems ranging from defining and processing information to evolution. Thus, the notion of information acquires novel understanding through its association with specific homeostatic pattern. The presence of a non-recursive component in that pattern renders any information symbol algorithmically unreachable from any other. In turn, the algorithmic uniqueness of any information symbol and any semantic unit makes every law in Nature unique and algorithmically uncompressible to a single universal law.

Another exclusive property of the non-recursivity of the relations structure-functionality is the substantiation of the semantic intelligence. The later implies that each and every complex system responds to environmental stimuli in a semantic-like manner whose properties are listed in the Introduction.

Further, the non-recursive relations structure-functionality provides a leading role of the hierarchy of functional organization of each complex system. In turn, it renders substantiation of general operational protocols for formation of scaling dependent and scaling independent moduli. Moreover, an exclusive property of the highly non-trivial interplay between both protocols is the coexistence of scaling dependent and scaling independent properties. It is worth noting that the formation of scaling dependent moduli and scaling independent fractals is an exclusive for the functional organization property. Thus, both moduli and fractals are functional objects and are not reducible in a recursive way to their structure.

Further, another exclusive property of the interplay between scaling dependent and scaling independent properties is the universality of the coarse-grained behavior of each and every complex system. Its exclusiveness culminates in its property to provide gradual changes of the specific mechanisms which bring about this universal behavior. The hallmark of this property is the scaling dependent exponent which provides the best fit of the fat tail in the distribution.

The universality of the coarse-grained behavior corresponds to the ubiquity of the empirical obtained distributions and thus it can serve as a hallmark for a novel evolutionary strategy available in an ever-changing environment. The major property of that strategy is the leading role of the non-extensive hierarchy of the functional organization which provides rapid functional adaptation of the behavior of corresponding system to the everchanging environment. The leading role of the functionality is best revealed by the separation of the response into two components: a specific individual one whose role is to provide fault tolerance of the response and the universal one aimed towards sustaining of the current homeostasis intact by means of restraining the diversity of the individual response to be within specific margins. This property constitutes its major difference with the traditional evolutionary strategy "survival of the fittest" which is the result of structural optimization supposed to provide best functioning in slowly changing environment. Thus, both evolution strategies become rather counterparts than opponents.

\section{References}

[1] West, G.B., Brown, J.H. and Enquist, B.J. (1997) Science, 276, 122-126. http://dx.doi.org/10.1126/science.276.5309.122

[2] Savage, V.M., Gillooly, J.F., Woodruff, W.H., West, G.B., Allen, A.P., Enquist, B.J. and Brown, J.H. (2004) Functional Ecology, 18, 257-282. http://dx.doi.org/10.1111/j.0269-8463.2004.00856.x

[3] Kauffmann, S. (1995) At Home in the Universe: The Search for the Laws of Self-organization and Complexity. Oxford University Press, New York-Oxford.

[4] Koleva, M.K. (2012) Boundedeness and Self-Organized Semantics: Theory and Applications. IGI-Global, Hershey.

[5] Koleva, M.K. (2014) Boundedeness and Self-Organized Semantics: On the Best Survival Strategy in an Ever Changing Environment. arXiv:1403.2583

[6] Feller, W. (1970) An Introduction to Probability Theory and Its Applications. John Willey \& Sons, New York. 